\title{
Postoperative apathy can neutralise benefits in quality of life after subthalamic stimulation for Parkinson's disease
}

\author{
Raul Martinez-Fernandez, ${ }^{1,2,3}$ Pierre Pelissier, ${ }^{1,2}$ Jean-Louis Quesada, 4,5 \\ Hélène Klinger, ${ }^{6,7,8}$ Eugénie Lhommée, ${ }^{1,2}$ Emmanuelle Schmitt, ${ }^{1,2}$ Valerie Fraix, ${ }_{1}^{1,2}$ \\ Stephan Chabardes, 1,2,9 Patrick Mertens, ${ }^{7,10}$ Anna Castrioto, ${ }^{1,2}$ Andrea Kistner, 1,2 \\ Emmanuel Broussolle, 6,7,8 Pierre Pollak, 1,2,11 Stéphane Thobois, 6,7,8 Paul Krack ${ }^{1,2}$
}

For numbered affiliations see end of article.

\section{Correspondence to} Paul Krack, Pavillon de Neurologie, CHU Grenoble, Grenoble Cedex 9 38043, France;

paul.krack@ujf-grenoble.fr

Received 17 December 2014 Revised 2 March 2015 Accepted 17 March 2015 Published Online First 1 May 2015

\section{CrossMark}

\section{To cite: Martinez-} Fernandez $R$, Pelissier $P$, Quesada J-L, et al. J Neurol Neurosurg Psychiatry 2016:87:311-318

\section{ABSTRACT}

Background Subthalamic nucleus deep brain stimulation (STN-DBS) improves motor symptoms of Parkinson's disease, leading to improvement in healthrelated quality of life (HRQoL). However, an excessive decrease in dopaminergic medication can lead to a withdrawal syndrome with apathy as the predominant feature. The present study aims to assess the impact of postoperative apathy on HRQoL.

Methods A cohort of 88 patients who underwent STNDBS was divided into two groups, those who were apathetic at 1 year and those who were not, as measured by the Starkstein scale. HRQoL was assessed using the Parkinson's disease questionnaire 39 (PDQ-39) and was compared between the two groups. We also compared activities of daily living, motor improvement and motor complications (Unified Parkinson's Disease Rating Scale, UPDRS), depression and anxiety, as well as cognition and drug dosages. Baseline characteristics and postoperative complications were recorded.

Results One year after surgery, $27.1 \%$ of patients suffered from apathy. While motor improvement was significant and equivalent in both the apathy $(-40.4 \%$ of UPDRS motor score) and non-apathy groups (-48.6\%), the PDQ-39 score did not improve in the apathy group $(-5.5 \% ; p=0.464)$, whereas it improved significantly $(-36.7 \% ; p \leq 0.001)$ in the non-apathy group. Change in apathy scores correlated significantly with change in HRQoL scores $(r=0.278, p=0.009)$. Depression and anxiety scores remained unchanged from baseline in the apathy group ( $p=0.409, p=0.075)$, while they improved significantly in patients without apathy $(p=0.006$, $p \leq 0.001)$. A significant correlation was found between changes in apathy and depression $(r=0.594, p \leq 0.001)$.

Conclusions The development of apathy after STN-DBS can cancel out the benefits of motor improvement in terms of HRQoL. Systematic evaluation and management of apathy occurring after subthalamic stimulation appears mandatory.

\section{INTRODUCTION}

Subthalamic nucleus deep brain stimulation (STN-DBS) is a well-established treatment for motor complications in Parkinson's disease (PD). ${ }^{1-5}$ Over the years, a large corpus of evidence has shown that STN-DBS improves health-related quality of life (HRQoL) in patients with $\mathrm{PD}^{3-12}$ to a larger extent than best medical treatment. ${ }^{3-5}$ Intriguingly, improvement in motor symptoms is not always accompanied by improvement in subjective well-being. ${ }^{6} 10 \quad 12 \quad 13$ Thus, while the doctor may be happy with the motor outcome, the patient and his/her relatives may complain that he/she is not benefiting fully from motor improvements. ${ }^{12}{ }^{14} \mathrm{HRQoL}$ is a measure of the patient's own judgement of their well-being. By assessing levels of satisfaction in a broad range of areas covering physical, mental and social aspects which may escape objective measurement, ${ }^{15} \mathrm{HRQoL}$ seems to be a more appropriate outcome measure than changes in motor status.

The relevance of non-motor symptoms in PD has been increasingly recognised over the past few decades. ${ }^{16}$ Neuropsychiatric aspects such as cognitive impairment, depression, anxiety and apathy have received particular attention because of their frequency and major impact on disability ${ }^{17}$ and HRQoL. ${ }^{16} 1819$ Apathy, which is common in PD (with a reported prevalence ranging from 20\% to $60 \%{ }^{1620} 21$ ), can occur in isolation or along with a depressive syndrome or dementia. ${ }^{21}$

The effects of chronic subthalamic stimulation on neuropsychiatric features in PD remain controversial. $^{22}$ While some authors report an improvement in apathy scores, ${ }^{23}$ others have noted no significant change after STN-DBS. ${ }^{4}$ However, there is growing evidence of apathy occurring or worsening after surgery. Apathy can occur several months after surgery and can develop as an isolated feature $^{24-30}$ or in association with depression and anxiety as part of a dopamine withdrawal syndrome. ${ }^{31}{ }^{32}$ In the long term, its development has been linked to dysexecutive syndrome in the context of disease progression. ${ }^{21}$ Despite its frequency, postoperative apathy has been largely neglected and the recent Earlystim study ${ }^{4}$ is the only randomised and controlled study of STN-DBS to have used a specific apathy assessment scale.

Our study aimed to discover whether the development of apathy after bilateral STN-DBS for PD impacts negatively on patient's HRQoL outcome independently of motor improvement.

\section{PATIENTS AND METHODS}

As surgical procedures, postsurgical management and outcome measures have all been reported 
previously. 3132 The ethics committee of Grenoble University approved the study and all patients gave informed written consent.

\section{Population and surgical procedures}

See figure 1 for the population flow chart. In a previous study, we performed a prospective analysis on the occurrence of apathy in the year following STN-DBS in patients operated on for PD in two centres (Grenoble and Lyon; see Thobois et al ${ }^{31}$ for selection criteria). Indication for surgery was based on current consensus. ${ }^{33}$ Postoperative management was part of a study protocol in which dopamine agonists were discontinued on the day of surgery. In the immediate postoperative period, levodopa treatment was reduced to the maximum extent permitted by improvement in motor state. We analysed the full database retrospectively in this study $(n=102$, patients included between 2005 and 2010) to examine the impact of apathy on HRQoL. Postoperative complications were recorded. We compare HRQoL changes 1 year after surgery in two subgroups from the whole sample followed in that study, separating patients with apathy from patients without apathy at that specific 12-month point.

\section{Clinical measures}

Clinical assessments took place in the month before surgery and at $12 \pm 1$ months after surgery. Cognitive assessment was performed at the 3-month postoperative follow-up. Patients completed the PD questionnaire 39 (PDQ-39) $^{34}$ at baseline and at 12 months to determine HRQoL. This 39-item, PD-specific selfassessment questionnaire measures HRQoL in daily life. It covers eight domains: mobility, activities of daily living, emotional well-being, stigma, social support, cognition, communication and bodily discomfort. The mean score of all domains (ie, the sum of all domain scores/8) provides an estimation of the overall impact of the disease on HRQoL through the PDQ-39SI (summary index score). Scores for each dimension and the PDQ-39SI range from 0 to 100, and lower scores indicate better-perceived HRQoL. Clinical evaluation of motor outcome was based on the Unified Parkinson's Disease Rating Scale (UPDRS) part III. Motor complications were measured using part IV of the UPDRS. The development of disabling motor symptoms known to have an impact on patients' HRQoL was recorded. Total levodopa dose at baseline and at 12-month follow-up was measured, and changes in levodopa and dopamine agonists were calculated as the total levodopa-equivalent daily dosage (LEDD). Overall cognitive status was assessed using the Mattis Dementia Rating Scale. Fronto-subcortical executive function was evaluated more specifically using the Frontal Assessment Battery. Chronic neuropsychiatric manifestations were studied through the Starkstein Apathy Scale for motivation, ${ }^{20}$ the Beck Depression Inventory (BDI) for depressive mood and the Beck Anxiety Inventory (BAI) for anxiety. Higher scores on these scales represent more severe symptoms. The Starkstein scale cut-off score for the diagnosis of apathy is $\geq 14$ out of a total of 42 points. Clinically relevant daily life nonmotor fluctuations were defined by a score $>2$ in summing 'non-motor ON' and 'non-motor OFF' from the Ardouin scale. ${ }^{32}$ The UPDRS part II activities of daily living were used to assess overall functional autonomy. At the 12-month evaluation, all scales were administered in the ON-stimulation, ON-medication condition (ie, chronic daily live state), except the UPDRS III, which was also administered in the ON-stimulation, OFF-medication state.

\section{Statistical analysis of clinical data}

Analysis was conducted using the per-protocol population. Data were summarised in terms of size and frequency for categorical parameters, and of means and SDs for continuous parameters, or by the median, 25 th and 75 th centiles when necessary. Baseline characteristics were compared using Student $t$ test for continuous parameters. A Mann-Whitney test was applied for non-Gaussian assumption. A paired Student $t$ test was used for continuous parameters, and a Wilcoxon test for non-Gaussian assumption. Independence between qualitative
Figure 1 Flow chart population analysis (PDQ-39SI, Parkinson's disease questionnaire 39 summary index).

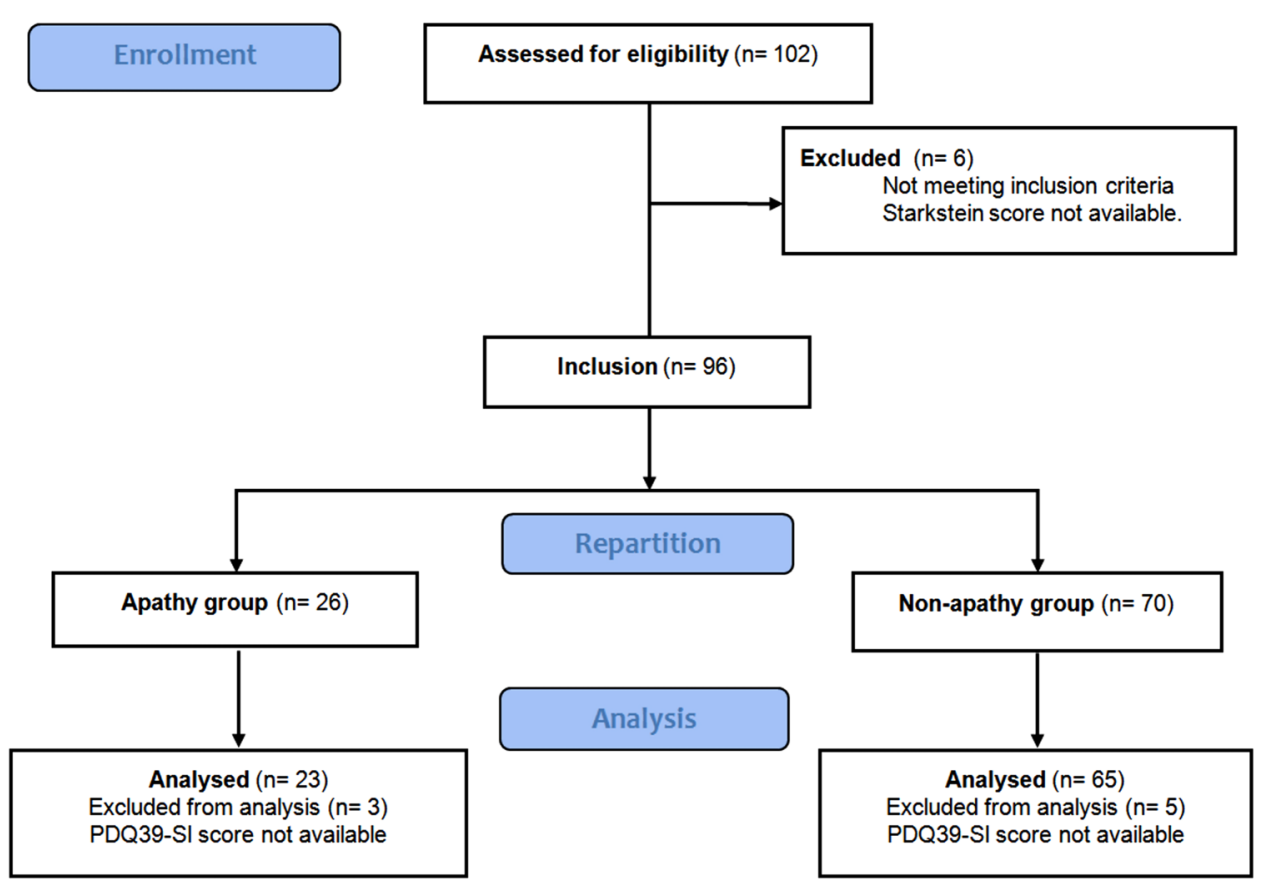


parameters was assessed using the $\chi^{2}$ test or Fisher's exact test. Correlation between quantitative parameters was assessed using the Pearson correlation test or Spearman when necessary. Partial correlation was used to adjust for confounding parameters. Main outcome PDQ-39SI between baseline and 1-year visit was tested using analysis of covariance analysis adjusted by baseline PDQ-39SI values to allow comparison of groups $(\mathrm{p}<0.05)$. Secondary (UPDRS III, PDQ-39 domains, BDI, BAI) outcomes were analysed using Student $\mathrm{t}$ test (or a Mann-Whitney test when necessary) to compare group effects between the baseline and T12 visit. Two multivariate models were conducted with the aim of verifying the predictive role of the Starkstein scale and the BDI on the PDQ-39. Statistical analyses were performed using STATA release V.12 (StataCorp, College Station, Texas, USA) PC-Software.

\section{RESULTS}

\section{Study population}

Ninety-six of the 102 patients had complete Starkstein scale data at baseline and at 12 months. Patients were divided into two groups at the 12-month follow-up point: 26 patients (27.1\%) who fulfilled apathy diagnosis criteria constituted the apathy group (AG) (table 1). Seventy patients (72.9\%) had apathy scores below 14 and were assigned to the non-apathy group (NAG). Eight patients (3 AG and 5 NAG) were excluded from the analysis due to the absence of PDQ-39 data. There were no statistical baseline differences between groups in terms of demographic characteristics, disease severity, PDQ-39SI score (including all domains, data not shown), motor score and motor complications. UPDRS II differed between groups but this difference disappeared at 12 months $(p=0.955)$. No patient suffered from apathy at baseline. However, the baseline mean score of the Starsktein scale was significantly higher in the AG. Preoperative BDI and BAI scores were equivalent in both groups. While the AG and NAG baseline Mattis scores showed no difference, patients from the AG had slightly lower frontal scores than patients from the NAG. However, neither scale showed any difference between groups at postoperative follow-up ( $p=0.138$ for the Mattis score, $p=0.223$ for the frontal score). Presence of daily life non-motor fluctuations was not significantly different between groups at baseline. There were no baseline differences between groups in dopaminergic medication.

\section{Medication changes}

Total levodopa dosage and total LEDD were markedly reduced and to the same extent in both groups (table 2). Total dopamine agonist equivalent reduction from baseline was also significant in both groups, with a lesser reduction in the AG.

\section{Motor and HRQoL outcome}

Both groups exhibited improvement in motor outcome at 12 months. UPDRS III improved significantly in the OFF-drug condition in both the AG $(-40.4 \%, \mathrm{p} \leq 0.001)$ and NAG $(-48.6 \%, \mathrm{p} \leq 0.001)$ with no difference between groups $(\mathrm{p}=0.446$; figure 2$)$. Severity and duration of dyskinesias and

Table 1 Demographic and clinical baseline characteristics of the whole sample, divided into the apathy and non-apathy groups

\begin{tabular}{|c|c|c|c|c|}
\hline & $\begin{array}{l}\text { Total population } \\
(\mathrm{n}=88)\end{array}$ & $\begin{array}{l}\text { Patients without apathy } \\
\text { at T12: non-apathy group }(n=65)\end{array}$ & $\begin{array}{l}\text { Patients with apathy } \\
\text { at T12: apathy group }(n=23)\end{array}$ & $p$ Value \\
\hline Age & $58.4(53.4 ; 63.8)$ & $58.2(54.2 ; 62.9)$ & $61.1(52.4 ; 64)$ & $0.680^{*}$ \\
\hline Duration of the disease & $10.6 \pm 3.2$ & $10.6 \pm 3.2$ & $10.7 \pm 3.2$ & $0.903 t$ \\
\hline Age at diagnosis & $47(42.5 ; 52.5)$ & $47(43 ; 52)$ & $48(42 ; 54)$ & $0.686^{*}$ \\
\hline Sex (\% male) & $43.2 \%(38)$ & $46.2 \%(30)$ & $34.8 \%(8)$ & $0.344 \ddagger$ \\
\hline PDQ-39SI & $68.8 \pm 27.4$ & $67.8 \pm 28.3$ & $71.3 \pm 25.3$ & $0.606 t$ \\
\hline UPDRS III (ON-medication) & $11.6 \pm 7.2$ & $11.3 \pm 7.4$ & $12.3 \pm 6.4$ & $0.578 \dagger$ \\
\hline UPDRS III (OFF-medication) & $38.7 \pm 12.5$ & $38.5 \pm 12.4$ & $39.1 \pm 13.1$ & $0.838 \dagger$ \\
\hline Activities of daily living UPDRS II (ON-medication) & $4(2 ; 7)$ & $3(1.5 ; 6.5)$ & $6(4 ; 8)$ & $0.022 \dagger$ \\
\hline Duration of dyskinesia & $1.56 \pm 0.97$ & $1.51 \pm 0.95$ & $1.70 \pm 1.02$ & $0.427^{*}$ \\
\hline Disability of dyskinesia & $1(1 ; 2)$ & $1(0 ; 2)$ & $1(0 ; 2)$ & $0.589+$ \\
\hline Duration of OFF periods & $1.60 \pm 0.74$ & $1.66 \pm 0.76$ & $1.43 \pm 0.66$ & $0.163 \dagger$ \\
\hline Starkstein scale (ON-medication) & $7(5 ; 9)$ & $7(4 ; 8)$ & $9(6 ; 11)$ & $0.020 \dagger$ \\
\hline BDI (ON-medication) & $8(5 ; 13)$ & $7.5(4 ; 13)$ & $9(6 ; 14)$ & $0.182 \dagger$ \\
\hline BAI (ON-medication) & $8(3 ; 15)$ & $7(2.5 ; 15)$ & $8(3 ; 12)$ & $0.839+$ \\
\hline Mattis Dementia Rating Scale & $140(138 ; 142)$ & $140(138 ; 143)$ & $139(137 ; 140)$ & $0.099+$ \\
\hline Frontal battery & $42.2(39 ; 47)$ & $43.6(40 ; 47)$ & $40.6(38.3 ; 42.9)$ & $0.038 \dagger$ \\
\hline Daily life non-motor fluctuations & $52.3 \%(46)$ & $50.8 \%(33)$ & $56.5 \%(13)$ & $0.635 \ddagger$ \\
\hline Total levodopa dose & $\begin{array}{l}962 \pm 425 \\
(0 ; 1980)\end{array}$ & $\begin{array}{l}928 \pm 401 \\
(0 ; 1800)\end{array}$ & $\begin{array}{l}1056 \pm 486 \\
(350 ; 1980)\end{array}$ & $0.218^{*}$ \\
\hline Total dopamine agonist equivalent dose & $\begin{array}{l}300(250 ; 400) \\
(0 ; 2160)\end{array}$ & $\begin{array}{l}300(250 ; 417) \\
(0 ; 2160)\end{array}$ & $\begin{array}{l}250(200 ; 360) \\
(0 ; 600)\end{array}$ & $0.207 \dagger$ \\
\hline Total levodopa+agonist equivalent & $\begin{array}{l}1298 \pm 473 \\
(300 ; 2660)\end{array}$ & $\begin{array}{l}1283 \pm 467 \\
(300 ; 2660)\end{array}$ & $\begin{array}{l}1339 \pm 498 \\
(533 ; 1900)\end{array}$ & $0.628^{*}$ \\
\hline Hoehn and Yahr ON stage & $2(1.5 ; 2)$ & $2(1.5 ; 2)$ & $2(2 ; 2.5)$ & $0.317 \dagger$ \\
\hline Hoehn and Yahr OFF stage & $3(2.5 ; 3)$ & $3(2.5 ; 3)$ & $2.5(2 ; 4)$ & $0.613 \dagger$ \\
\hline $\begin{array}{l}\text { Average } \pm S D \text {, median }(25 \text { th; } 75 \text { th centiles). } \\
\text { Figures in bold indicate that the } p \text { value is statistically, } \\
\text { *Student } t \text { test. } \\
\text { †Mann-Whitney test. } \\
\ddagger \chi^{2} \text { Test. } \\
\text { BAl, Beck Anxiety Inventory; BDI, Beck Depression In }\end{array}$ & ificant. & & & \\
\hline
\end{tabular}


Table 2 Medical treatment at baseline and 12-month follow-up (T12) of both groups and comparison in dosage reduction between groups

\begin{tabular}{|c|c|c|c|c|c|c|}
\hline & \multicolumn{3}{|c|}{ Non-apathy group $(n=65)$} & \multicolumn{3}{|c|}{ Apathy group $(n=23)$} \\
\hline & Baseline & T12 & p Value & Baseline & $\mathrm{T} 12$ & p Value \\
\hline Total daily levodopa dose & $\begin{array}{l}928 \pm 401 \\
900(628 ; 1200)\end{array}$ & $\begin{array}{l}291 \pm 330 \\
200(75 ; 400)\end{array}$ & $\leq 0.001^{*}$ & $\begin{array}{l}1056 \pm 486 \\
927(626 ; 1383)\end{array}$ & $\begin{array}{l}399 \pm 446 \\
200(75 ; 600)\end{array}$ & $\leq 0.001 *$ \\
\hline Total daily dopamine agonist equivalent dose & $\begin{array}{l}355 \pm 337 \\
300(250 ; 417)\end{array}$ & $\begin{array}{l}85 \pm 154 \\
0(0 ; 100)\end{array}$ & $\leq 0.001^{*}$ & $\begin{array}{l}283 \pm 130 \\
250(200 ; 360)\end{array}$ & $\begin{array}{l}159 \pm 203 \\
0(0 ; 333)\end{array}$ & $0.017^{*}$ \\
\hline Total daily levodopa equivalents & $\begin{array}{l}1283 \pm 467 \\
1252(967 ; 1550)\end{array}$ & $\begin{array}{l}375 \pm 346 \\
300(83 ; 550)\end{array}$ & $\leq 0.001^{*}$ & $\begin{array}{l}1339 \pm 498 \\
1267(917 ; 1750)\end{array}$ & $\begin{array}{l}553 \pm 489 \\
333(200 ; 900)\end{array}$ & $\leq 0.001 *$ \\
\hline \multicolumn{6}{|l|}{ Comparison between groups at 12 months } & $\mathrm{p}$ Value \\
\hline \multicolumn{2}{|l|}{ Total daily levodopa dose reduction } & \multicolumn{2}{|l|}{$\begin{array}{l}-637 \pm 505 \\
(-69 \%)\end{array}$} & \multicolumn{2}{|l|}{$\begin{array}{l}-657 \pm 562 \\
(-62 \%)\end{array}$} & $0.878 \dagger$ \\
\hline \multicolumn{2}{|c|}{ Total daily dopamine agonist equivalent dose reduction } & $\begin{array}{l}-270 \pm 388(-76 \%) \\
-267(-375 ;-67)\end{array}$ & & \multicolumn{2}{|l|}{$\begin{array}{l}-124 \pm 225(-44 \%) \\
-200(-250 ; 100)\end{array}$} & $0.036 \ddagger$ \\
\hline \multicolumn{2}{|l|}{ Total daily levodopa equivalents reduction } & $\begin{array}{l}-908 \pm 594 \\
(-71 \%)\end{array}$ & & \multicolumn{2}{|l|}{$\begin{array}{l}-786 \pm 603 \\
(-59 \%)\end{array}$} & $0.401 \dagger$ \\
\hline
\end{tabular}

Average \pm SD, median (25th; 75th centiles).

Figures in bold indicate that the $p$ value is statistically significant.

*Wilcoxon-test.

tStudent $t$ test.

$\ddagger$ Mann-Whitney test.

OFF-period duration had improved significantly in both groups at 12 months $(\mathrm{p} \leq 0.001)$, and there was no difference between groups at the final follow-up. While PDQ-39SI decreased in the
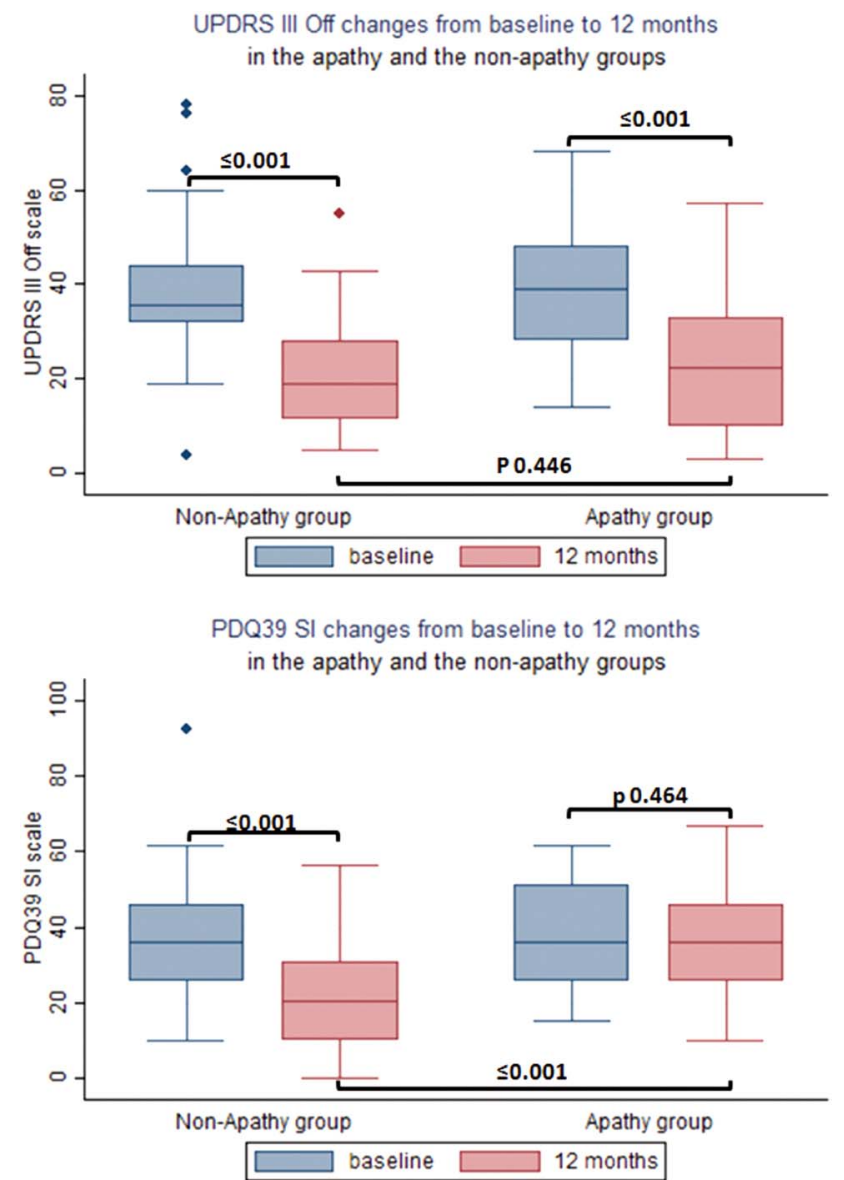

Figure 2 Changes in the scores of the Unified Parkinson's Disease Rating Scale (UPDRS) III OFF-medication and in the Parkinson's disease questionnaire 39 summary index (PDQ-39SI) from a preoperative condition to 12-month follow-up in the apathy and non-apathy groups.
AG by a non-significant $\quad-5.5 \% \quad(-2 \pm 12.9, \quad \mathrm{p}=0.464)$, a $-36.7 \%(-12.8 \pm 17.2, p \leq 0.001)$ amelioration occurred in the NAG and the difference between groups was statistically different at 12 months $(p \leq 0.001$; figure 2). Furthermore, changes in apathy scores between baseline and final outcome for the whole sample correlated significantly with changes in PDQ-39SI $(\mathrm{r}=0.28, \mathrm{p}=0.009)$ in the $\mathrm{ON}$-drug state, even when controlling for motor state, anxiety and activities of daily living. Significance was lost when controlling for depression. A low correlation was observed between UPDRS III and PDQ-39SI scores at 12 months in the $\mathrm{ON}$-medication condition $(\mathrm{r}=0.25$, $\mathrm{p}=0.021$ ); however, they did not correlate significantly in the OFF-medication state. The first multivariate model, incorporating the BDI, UPDRS II, III and IV and reduction in LEDD as explanatory factors, demonstrates that the BDI and UPDRS III were predictors of impairment in HRQoL $(p=0.130$ and $\mathrm{p}=0.046$, respectively). The second model, incorporating the Starkstein score, UPDRS II, III and IV and reduction in LEDD as explanatory factors, showed the Starkstein score to be a predictor of impairment in HRQoL $(p=0.103)$.

\section{PDQ-39 dimensions}

In the NAG, five PDQ-39 dimensions improved significantly from the preoperative condition (mobility, activities of daily living, stigma, cognition and bodily discomfort). In the AG, stigma was the only domain which improved significantly compared with baseline (table 3). Statistically significant differences in outcome appeared between groups for mobility $(-17.6 \%$ improvement in the NAG vs $6.1 \%$ worsening in the $A G$, $\mathrm{p} \leq 0.001)$ and communication $(0.3 \%$ impairment in the NAG vs $11.2 \%$ worsening in the $\mathrm{AG}, \mathrm{p}=0.044$; figure 3 ).

\section{Depression and anxiety outcomes}

The NAG BDI and BAI scores in the ON-medication condition improved significantly compared with baseline. The AG showed a slight impairment in the BDI and a non-significant improvement in the BAI between baseline and final follow-up. The change from baseline $(\delta)$ between groups was significantly different for the BDI $(p=0.043)$ but not for the BAI $(p=0.384$; figure 4). A significant correlation between the change in the 
Table 3 Modification of PDQ-39 domains between baseline and 12-month follow-up (T12) in the apathy and non-apathy groups. Lower scores represent a better subjective perception of quality of life expressed in a range from 0 to 100

\begin{tabular}{|c|c|c|c|c|c|c|c|}
\hline & \multirow[b]{2}{*}{ Mean value* } & \multicolumn{3}{|c|}{ Non-apathy group $(n=65)$} & \multicolumn{3}{|c|}{ Apathy group $(n=23)$} \\
\hline & & Baseline & $\mathrm{T} 12$ & p Value & Baseline & $\mathrm{T} 12$ & $\mathrm{p}$ Value \\
\hline Mobility $(n=63 ; n=21)$ & $42.95 \pm 28.43$ & $42.4 \pm 20.4$ & $24.8 \pm 20.1$ & $\leq 0.001 \dagger$ & $41.1 \pm 20.5$ & $47.3 \pm 24.5$ & $0.182 \dagger$ \\
\hline Activities of daily living ( $n=65 ; n=22$ ) & $38.94 \pm 24.76$ & $40.3 \pm 18.1$ & $22.1 \pm 19$ & $\leq 0.001 \dagger$ & $41.9 \pm 17.2$ & $34.5 \pm 24.5$ & $0.169 t$ \\
\hline Emotional well-being $(n=62 ; n=22)$ & $37.92 \pm 21.05$ & $28.3 \pm 19.7$ & $23.3 \pm 22$ & $0.076 \ddagger$ & $31.3 \pm 21$ & $34.9 \pm 19.3$ & $0.447 \dagger$ \\
\hline Stigma $(n=64 ; n=23)$ & $27.54 \pm 23.17$ & $32 \pm 23.3$ & $18.3 \pm 19.7$ & $\leq 0.001 \dagger$ & $36.6 \pm 23.6$ & $24.9 \pm 18.4$ & $0.049+$ \\
\hline Social support $(n=59 ; n=23$ ) & $14.78 \pm 18.08$ & $10.1 \pm 15.4$ & $9.1 \pm 16.1$ & $0.297 \ddagger$ & $12.9 \pm 17$ & $17.8 \pm 22.3$ & $0.256 \ddagger$ \\
\hline Cognition $(n=64 ; n=21)$ & $33.03 \pm 20.35$ & $23.1 \pm 17.9$ & $14.7 \pm 16$ & $\leq 0.001 \ddagger$ & $27.7 \pm 16.5$ & $25.3 \pm 16.2$ & $0.465 t$ \\
\hline Communication $(n=63 ; n=23)$ & $27.99 \pm 24.19$ & $27.7 \pm 18.2$ & $27.9 \pm 19.5$ & $0.917 \ddagger$ & $26.8 \pm 22.2$ & $38 \pm 23.9$ & $0.055 t$ \\
\hline Bodily discomfort $(n=64 ; n=22)$ & $40.91 \pm 24.07$ & $41.3 \pm 21.9$ & $24.8 \pm 20.6$ & $\leq 0.001 \ddagger$ & $43.5 \pm 25.5$ & $30.4 \pm 15.3$ & $0.051 \ddagger$ \\
\hline \multicolumn{8}{|c|}{$\begin{array}{l}\text { Average } \pm \text { SD. } \\
\text { Figures in bold indicate that the } p \text { value is statistically significant. } \\
\text { *From the study of validity of the PDQ-39, on a sample of patients with a mean age of } 69.8 \text { years (range 34-90). } \\
\text { †Student } t \text { test. } \\
\text { †Wilcoxon test. }\end{array}$} \\
\hline
\end{tabular}

PDQ-39SI score and that of the BDI was found $(r=0.232$, $\mathrm{p}=0.033)$. No correlation was observed between the BAI and PDQ-39SI changes $(r=0.128, p=0.243)$. Finally, there was a strong correlation between changes in the Starkstein scale and the BDI $(r=0.594, \mathrm{p} \leq 0.001)$.

\section{Surgical complications and postoperative side effects}

From the 88 selected patients, one suffered a symptomatic cerebral haemorrhage. Reoperation was needed because of electrode misplacement $(n=1)$, electrode migration $(n=1)$ and cable fracture $(n=1)$. In one patient, an infection led to the removal of subcutaneous material and electrodes. Four patients had transient symptomatic contusions along the electrode trajectory (psychosis $n=1$, somnolence $n=1$, stereotypies $n=1$, confusion $\mathrm{n}=1$ ). Other transient side effects not associated with brain lesions were postoperative behavioural or cognitive disturbances ( psychosis $n=3$, hallucination $n=2$, confusion $n=4$ ) and transient hypomania after dopamine agonist reintroduction to treat restless legs syndrome $(n=1)$. Worsening of restless legs syndrome was reported by six patients. Other reported transient general side effects were: urinary retention $n=1$, incontinence $n=1$, blurred vision $n=1$, gastroenteritis $n=1$, dysuria $n=1$ and pneumonia with transient cardiorespiratory arrest in the context of a postoperative akinetic crisis $n=1$. Non-transient side effects included speech $(n=20)$ and swallowing disturbances $(n=1)$. The walking and balance worsened in 11 patients. In six patients, pre-existing freezing of gait worsened. Five patients complained of pain. One patient exhibited persistent hypotension with postoperative onset.

\section{DISCUSSION}

One year after surgery, $27.1 \%$ of the present cohort of patients whose postoperative dopaminergic treatment had been considerably reduced presented with apathy, measured by the Starkstein scale. While motor improvement was significant and equivalent in the AG and NAG $(-40.4 \%$ vs $-48.6 \%)$, the PDQ-39 score did not improve in the AG, whereas a significant improvement emerged in the NAG (figure 2). Stigma was the only PDQ-39 dimension that improved in the AG, whereas in the NAG mobility, activities of daily living, stigma, cognition and bodily discomfort domains improved. Changes from baseline to 12-month follow-up in apathy and quality of life scores correlated significantly, but significance was lost when controlling for depression. Depression and anxiety scores remained unchanged from baseline in the AG, but improved significantly in the NAG (figure 4). Depression scores were equivalent between groups at
Figure 3 Comparison of the modifications $(\delta)$ in PDQ-39 subscores from baseline to 12-month follow-up between the apathy and non-apathy groups. $p$ Values of the difference of the change between groups are detailed above each column. The red arrow (positive values) indicates worsening whereas the green arrow (negative values) represents alleviation. $A D L$, activities of daily living; PDQ-39SI, Parkinson's disease questionnaire 39 summary index.

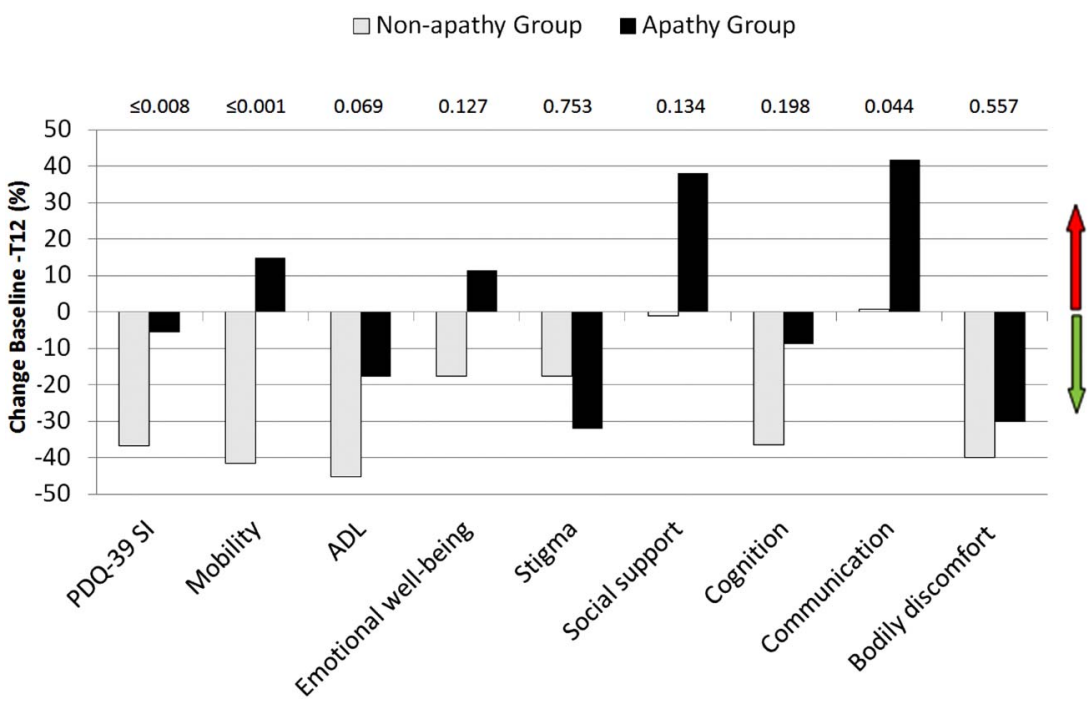



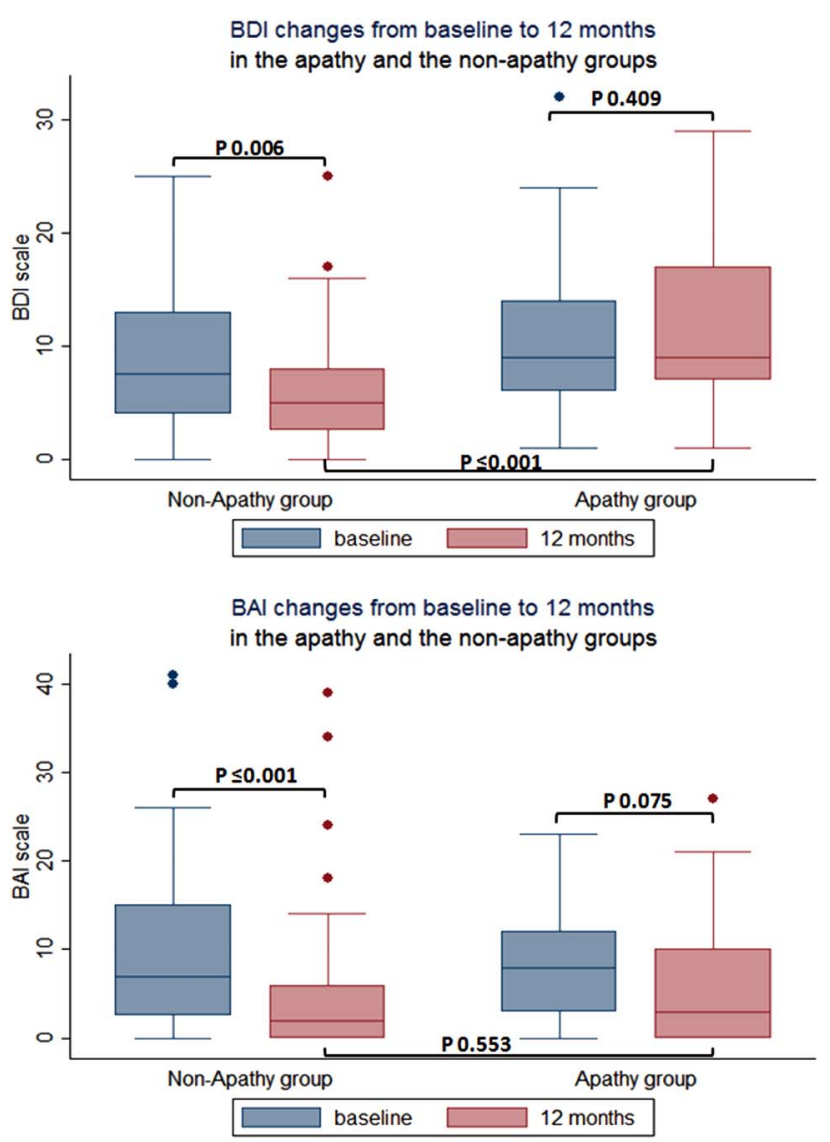

Figure 4 Beck Depression Inventory (BDI) and Beck Anxiety Inventory (BAI) changes from baseline (in ON-medication condition) to 12 months (in ON-medication/ON-stimulation state, ie, daily living situation) in the apathy and non-apathy groups. For both scales, higher scores represent more severe symptoms (in a range from 0 to 63).

baseline but at 12 months they differed significantly. Changes in the Starkstein scale and BDI scores correlated significantly. Multiple regression analysis revealed that apathy and depression were independent predictors of HRQoL changes.

So far, only a few specific clinical or demographic features have been associated with HRQoL outcome in patients with PD STN-DBS; ${ }^{7-9} 13$ this study is the first to clearly demonstrate the negative impact of apathy on HRQoL in a postsurgical PD series.

\section{Prevalence of postoperative apathy}

Data are scarce on the prevalence of apathy in the early postoperative period, with a range from $23 \%$ to $60 \% .^{25} 28313536$ In addition, several other groups have reported overall increases in mean apathy scores after STN-DBS. ${ }^{46-30}$ Data on long-term apathy occurrence in patients with STN-DBS are even more limited, with two works reporting rates of $12 \%{ }^{2}$ and $4.3 \% \%^{37}$ at 5 years. Several reasons explain the disparity in the prevalence of STN-DBS-related apathy. Apathy can present as two different entities with specific pathological origins and singular therapeutic approaches. ${ }^{21}$ First, in some patients, early postoperative apathy corresponds to a dopaminergic withdrawal syndrome triggered by a postoperatory decrease in dopaminergic medication which unmasks presynaptic degeneration of mesolimbic dopaminergic terminals. ${ }^{31}$ This situation mainly explains apathy in the first year of follow-up. The prevalence of this early postoperative apathy also depends on the management of drug and stimulation parameters. ${ }^{21} 22{ }^{36}$ In our study, total reduction in dopaminergic treatment was equivalent between groups, but this does not exclude occurrence of the dopamine withdrawal syndrome. Indeed, apathy is frequent in general PD but not present in all patients. As aforementioned, individual differences in the neurodegenerative process, particularly more widespread mesolimbic dopaminergic denervation, predispose to non-motor withdrawal symptoms with apathy being the most prominent symptom. ${ }^{31}$ Postoperative-withdrawal apathy may occur up to 12 months after surgery $^{31}$ due to the slowly progressive mechanisms of desensitisation. ${ }^{38}$ Therefore, in some of the patients of this study, apathy at 12 months may represent apathy of recent onset not yet successfully treated. Second, a more delayed dopa-resistant apathy as part of the development of a dysexecutive syndrome related to the progression of $\alpha$-synucleinopathy can occur during long-term postoperative follow-up, whatever the surgical target. ${ }^{21} 39$

Our study does not correspond to regular clinical practice as patients were included in a protocol requiring complete preoperative withdrawal of dopamine agonists, and their reintroduction only if apathy developed during monthly screening. However, levodopa was reduced based on stimulation-induced improvement on the patients' motor condition, a modus operandi that is usually applied when dealing with immediate postsurgical therapeutic adjustment in PD. Thus, even if our reported apathy prevalence might be higher than the usual, the development of apathy does not come as a surprise in regular clinical practice.

\section{Phenotype of the hypodopaminergic patient after STN-DBS} Patients in the AG had higher anxiety and depression scores than those in the NAG. It has long been established that anxiety and depression are highly prevalent in PD. ${ }^{18}$ Although apathy has been recognised as a prominent feature of $\mathrm{PD},{ }^{21}$ these two entities have generally attracted more attention than apathy. ${ }^{18}$ However, apathy is about twice as frequent as depression after subthalamic surgery, while PD depression is systematically accompanied by apathy. ${ }^{31}$ Therefore, we advocate that apathy in patients with PD treated with STN-DBS requires systematic screening, as it constitutes the core and the 'tip of the iceberg' of a larger hypodopaminergic syndrome which also includes anxiety, depression ${ }^{31}$ and pain. ${ }^{40}$

\section{Lessons for the management of postoperative apathy}

Postoperative non-motor symptoms can potentially be modified by optimising drug management. The Earlystim study supports the importance of clinical management in preventing the occurrence of apathy after STN-DBS by showing that surgical patients with optimal medical treatment do not display higher levels of apathy than non-operated patients with best medical management in a 2-year follow-up period. ${ }^{4}$ Considering the findings of our study, if postoperative apathy presents, dopaminergic drugs should be reintroduced or increased as the patient's subjective well-being should prevail as the primary goal over the physicians' and caregivers' desire to see the patient completely free from dyskinesia, which may not bother the patient himself. Patients with more marked preoperative non-motor fluctuations require particular attention as their presence represents a risk factor for dopamine withdrawal syndrome. ${ }^{31}$

Clinical features of the hypodopaminergic syndrome overlap, and we found that depression scores also correlated with those of apathy and the PDQ-39SI. Therefore, when a hypodopaminergic state is encountered, it should be treated globally because the development of any one of its features can have an impact 
on HRQoL. Consequently, these symptoms must be screened for and managed as carefully as motor signs, especially since they are potentially reversible with medical treatment, and improvement in HRQoL ensues. ${ }^{36}$ Importantly, while hypodopaminergic syndrome occurrence may be particularly relevant in the first year after STN-DBS, this context serves as a model that can also be generalised to the whole non-demented PD population.

\section{Changes in PDQ-39 domains}

The difference between the AG and the NAG in terms of PDQ-39 domain changes was greatest for mobility and communication. A close scrutiny of the PDQ-39 questions which assess mobility reveals that they also evaluate goal-directed motor behaviour, which is impaired by apathy. Differences in communication cannot be attributed to 'motor' dysarthria as no differences were found between groups in the UPDRS item assessing speech (data not shown). Reduction in verbal fluency, which may contribute to a decline in communication skills, is frequently reported after STN-DBS. ${ }^{11}{ }^{41}$ In patients with apathy, this can probably be aggravated by a lack of autoactivation which reduces the motivation to initiate conversation.

Stigma and bodily discomfort were the only PDQ-39 domains which improved in the AG to virtually the same extent as in the NAG. Improvement in the more visible motor symptoms that result in social handicap, such as tremor or dyskinesia, can explain the improvement in stigma perceived by the patients. Pain, which is one of the major symptoms accounting for bodily discomfort, has both motor and non-motor origins ${ }^{42}$ and responds to STN-DBS. ${ }^{43}$ The amelioration of motor complications and the relief in pain resulted in improved bodily comfort in both groups.

\section{Limitations}

Apathy impacts HRQoL independently. However, the low intensity of the correlation between the Starkstein scale and PDQ-39SI scores at 12 months when controlling for depression should make us consider the possibility that depression also influences the HRQoL outcome. Improvement could therefore be limited if only apathy is treated. It is well known that depression in PD is detrimental to HRQoL. ${ }^{18}$ As previously mentioned, although apathy and depression are different conditions, there is some overlap in clinical symptoms and pathophysiological mechanisms, ${ }^{31} 32$ and indeed we found a strong correlation between changes in apathy and depression scores. Treatment of apathy with a dopamine agonist also had a beneficial impact on depression. ${ }^{36}$

At baseline, apathy scores were higher in the AG than in the NAG. Even if motivation is not a dichotomous condition, a two-point difference below the cut-off for the diagnosis of apathy in a 42-point scale should not have sufficient clinical significance to impact HRQoL.

\section{CONCLUSION}

The results of this study show that occurrence of postoperative apathy in patients with PD who have undergone STN-DBS can independently counteract the beneficial effects of motor improvement in terms of HRQoL. Apathy can present after STN-DBS for PD depending on clinical management and individual, patient-related risk factors. We have shown that its development is closely linked to a worsening in self-reported quality of life despite surgical success from a motor viewpoint. The main purpose of functional neurosurgery for PD should be to improve patients' quality of life. Therefore, notwithstanding the physician's satisfaction with the more eye-catching amelioration in motor features, in order to render functional neurosurgery meaningful, apathy must be systematically screened for using specific scales due to its major impact on HRQoL. This type of systematic approach is of particular interest because the reintroduction of the dopamine agonist has proven to be effective for postoperative apathy. ${ }^{36}$ Finally, since apathy in PD is largely related to presynaptic mesolimbic dopaminergic denervation, our findings can also be extrapolated to the general nondemented PD population where the same attention to apathy is also required for optimal patient management.

\section{Author affiliations}

${ }^{1}$ Movement Disorders Unit, Department of Psychiatry and Neurology, CHU de Grenoble, Université de Grenoble Alpes, Grenoble, France

${ }^{2}$ INSERM, U386, Grenoble Institut de Neurosciences, Grenoble, France

${ }^{3}$ CINAC-HM Puerta del Sur, CEU-San Pablo University, Madrid, Spain

${ }^{4}$ Centre d'Investigation Clinique, CHU de Grenoble, Univesité Joseph Fourier, Grenoble, France

${ }^{5}$ Direction de la recherche clinique, Département scientifique, CHU Grenoble, Grenoble, France

${ }^{6}$ Hospices Civils de Lyon, Hôpital Neurologique, Neurologie C, Lyon, France ${ }^{7}$ Faculté de Médecine et de Maïeutique Lyon Sud Charles Mérieux, Université de Lyon 1, Lyon, France

${ }^{8}$ CNRS, UMR 5229, Centre de Neurosciences Cognitives, Bron, France

${ }^{9}$ Deparment of Neurosurgery, CHU de Grenoble, Grenoble, France

${ }^{10}$ Hospices Civils de Lyon, Hôpital Neurologique, Neurochirurgie A, Lyon, France

${ }^{11}$ Service de Neurologie, Hôpitaux Universitaires de Genève, Geneva, Switzerland

Acknowledgements The authors thank Mrs Cate Dal Molin for English language correction.

Contributors RM-F, PPe and J-LQ contributed to the design of the work, analysis and interpretation of data. HK, ES, VF, SC, PM, AK, PPo and ST contributed to the design of the work, acquisition and interpretation of data. EL and PK contributed to the design of the work, acquisition, analysis and interpretation of data. $A C$ and $E B$ contributed to the design of the work and interpretation of data. RM-F wrote the first draft and revised it critically. All the authors revised the subsequent drafts critically. All authors gave final approval of the version published.

Funding This work was supported by a grant from the Programme Hospitalier de Recherche Clinique Interregional and Servier.

Competing interests SC, PPo, PK, PM and ST were granted funds from Medtronic for research purposes in the field of deep brain stimulation. PK was granted funds from St. Jude for research purposes in the field of deep brain stimulation and received research support from France Parkinson, INSERM (French National Institute of Health and Research in Medicine), Edmond J.\& Lily Safra Foundation, Grenoble University Hospital, Orkyn, Novartis, UCB, LVL, Boston Scientific. AC was granted funds from Edmond J.\& Lily Safra Foundation. Several authors received reimbursement of travel expenses to scientific meetings or honoraria for lecturing or consultation from Medtronic (SC, PPo, PK, ST, EB and PM), Euthérapie Company (PPo, PK, ST and EB), Abvie (EB), Novartis Pharma (PK and EB), UCB (RM-F and PK), St. Jude (RM-F and PK), Lundbeck (RM-F and PK), Boehringer Ingelheim (PK), Orkyn (PK), Abbott (PK), Orion (PK), TEVA (PK) and Boston Scientific (PK).

Patient consent Obtained.

Ethics approval The ethics committee of Grenoble University.

Provenance and peer review Not commissioned; externally peer reviewed.

\section{REFERENCES}

1 Limousin $\mathrm{P}$, Krack $\mathrm{P}$, Pollak $\mathrm{P}$, et al. Electrical stimulation of the subthalamic nucleus in advanced Parkinson's disease. N Engl J Med 1998;339:1105-11.

2 Krack P, Batir A, Van Blercom N, et al. Five-year follow-up of bilateral stimulation of the subthalamic nucleus in advanced Parkinson's disease. N Engl J Med 2003:349:1925-34.

3 Deuschl G, Schade-Brittinger C, Krack P, et al. A randomized trial of deep-brain stimulation for Parkinson's disease. N Engl J Med 2006;355:896-908.

4 Schuepbach WM, Rau J, Knudsen K, et al. Neurostimulation for Parkinson's disease with early motor complications. N Engl J Med 2013;368:610-22.

5 Weaver FM, Follett K, Stern M, et al. Bilateral deep brain stimulation vs best medical therapy for patients with advanced Parkinson disease: a randomized controlled trial. JAMA 2009;301:63-73.

6 Just $H$, Ostergaard K. Health-related quality of life in patients with advanced Parkinson's disease treated with deep brain stimulation of the subthalamic nuclei. Mov Disord 2002;17:539-45. 
7 Lagrange E, Krack P, Moro E, et al. Bilateral subthalamic nucleus stimulation improves health-related quality of life in PD. Neurology 2002;59:1976-8.

8 Martinez-Martin P, Valldeoriola F, Tolosa E, et al. Bilateral subthalamic nucleus stimulation and quality of life in advanced Parkinson's disease. Mov Disord 2002;17:372-7.

9 Troster Al, Fields JA, Wilkinson S, et al. Effect of motor improvement on quality of life following subthalamic stimulation is mediated by changes in depressive symptomatology. Stereotact Funct Neurosurg 2003;80:43-7.

10 Gronchi-Perrin A, Viollier S, Ghika J, et al. Does subthalamic nucleus deep brain stimulation really improve quality of life in Parkinson's disease? Mov Disord 2006;21:1465-8.

11 Witt K, Daniels C, Reiff J, et al. Neuropsychological and psychiatric changes after deep brain stimulation for Parkinson's disease: a randomised, multicentre study. Lancet Neurol 2008;7:605-14.

12 Maier $\mathrm{F}$, Lewis CJ, Horstkoetter $\mathrm{N}$, et al. Patients' expectations of deep brain stimulation, and subjective perceived outcome related to clinical measures in Parkinson's disease: a mixed-method approach. J Neurol Neurosurg Psychiatry 2013:84:1273-81.

13 Witt K, Daniels C, Krack P, et al. Negative impact of borderline global cognitive scores on quality of life after subthalamic nucleus stimulation in Parkinson's disease. J Neurol Sci 2011;310:261-6.

14 Agid Y, Schupbach M, Gargiulo M, et al. Neurosurgery in Parkinson's disease: the doctor is happy, the patient less so? J Neural Transm Supp/ 2006;70:409-14.

15 Meyers AR, Gage H, Hendricks A. Health-related quality of life in neurology. Arch Neurol 2000;57:1224-7.

16 Martinez-Martin P, Rodriguez-Blazquez C, Kurtis MM, et al. The impact of non-motor symptoms on health-related quality of life of patients with Parkinson's disease. Mov Disord 2011;26:399-406.

17 Weintraub D, Moberg PJ, Duda JE, et al. Effect of psychiatric and other nonmotor symptoms on disability in Parkinson's disease. J Am Geriatr Soc 2004;52:784-8.

18 Schrag A. Quality of life and depression in Parkinson's disease. J Neurol Sci 2006:248:151-7.

19 Barone P, Antonini A, Colosimo C, et al. The PRIAMO study: a multicenter assessment of nonmotor symptoms and their impact on quality of life in Parkinson's disease. Mov Disord 2009;24:1641-9.

20 Starkstein SE, Mayberg HS, Preziosi TJ, et al. Reliability, validity, and clinical correlates of apathy in Parkinson's disease. J Neuropsychiatry Clin Neurosci 1992;4:134-9.

21 Pagonabarraga J, Kulisevsky J, Strafella A, et al. Apathy in Parkinson's disease: a model to study motivated behaviours. Lancet Neurol 2015 Apr 13. In press.

22 Castrioto $A$, Lhommee $E$, Moro $E$, et al. Mood and behavioural effects of subthalamic stimulation in Parkinson's disease. Lancet Neurol 2014;13:287-305.

23 Campbell MC, Black KJ, Weaver PM, et al. Mood response to deep brain stimulation of the subthalamic nucleus in Parkinson's disease. J Neuropsychiatry Clin Neurosci 2012;24:28-36.

24 Funkiewiez A, Ardouin C, Caputo E, et al. Long term effects of bilateral subthalamic nucleus stimulation on cognitive function, mood, and behaviour in Parkinson's disease. J Neurol Neurosurg Psychiatry 2004;75:834-9.

25 Drapier D, Drapier S, Sauleau P, et al. Does subthalamic nucleus stimulation induce apathy in Parkinson's disease? J Neurol 2006;253:1083-91.
26 Le Jeune $F$, Drapier $D$, Bourguignon $A$, et al. Subthalamic nucleus stimulation in Parkinson disease induces apathy: a PET study. Neurology 2009;73:1746-51.

27 Kirsch-Darrow L, Zahodne LB, Marsiske $M$, et al. The trajectory of apathy after deep brain stimulation: from pre-surgery to 6 months post-surgery in Parkinson's disease. Parkinsonism Relat Disord 2011;17:182-8.

28 Funkiewiez A, Ardouin C, Cools R, et al. Effects of levodopa and subthalamic nucleus stimulation on cognitive and affective functioning in Parkinson's disease. Mov Disord 2006;21:1656-62.

29 Drapier D, Peron J, Leray E, et al. Emotion recognition impairment and apathy after subthalamic nucleus stimulation in Parkinson's disease have separate neural substrates. Neuropsychologia 2008;46:2796-801.

30 Porat $\mathrm{O}$, Cohen OS, Schwartz $\mathrm{R}$, et al. Association of preoperative symptom profile with psychiatric symptoms following subthalamic nucleus stimulation in patients with Parkinson's disease. I Neuropsychiatry Clin Neurosci 2009:21:398-405.

31 Thobois S, Ardouin C, Lhommee E, et al. Non-motor dopamine withdrawal syndrome after surgery for Parkinson's disease: predictors and underlying mesolimbic denervation. Brain 2010;133(Pt 4):1111-27.

32 Lhommee $\mathrm{E}$, Klinger $\mathrm{H}$, Thobois $\mathrm{S}$, et al. Subthalamic stimulation in Parkinson's disease: restoring the balance of motivated behaviours. Brain 2012; 135(Pt 5):1463-77.

33 Pollak P. Deep brain stimulation for Parkinson's disease - patient selection. Handb Clin Neurol 2013;116:97-105.

34 Peto V, Jenkinson C, Fitzpatrick $R$, et al. The development and validation of a short measure of functioning and well being for individuals with Parkinson's disease. Qual Life Res 1995;4:241-8.

35 Czernecki V, Schupbach M, Yaici S, et al. Apathy following subthalamic stimulation in Parkinson disease: a dopamine responsive symptom. Mov Disord 2008;23:964-9.

36 Thobois $\mathrm{S}$, Lhommee $\mathrm{E}$, Klinger $\mathrm{H}$, et al. Parkinsonian apathy responds to dopaminergic stimulation of D2/D3 receptors with piribedil. Brain 2013;136(Pt 5):1568-77.

37 Gervais-Bernard H, Xie-Brustolin J, Mertens P, et al. Bilateral subthalamic nucleus stimulation in advanced Parkinson's disease: five year follow-up. J Neurol 2009;256:225-33.

38 Castrioto A, Kistner A, Klinger $H$, et al. Psychostimulant effect of levodopa: reversing sensitisation is possible. J Neurol Neurosurg Psychiatry 2013;84:18-22.

39 Rodriguez-Oroz MC, Moro E, Krack P. Long-term outcomes of surgical therapies for Parkinson's disease. Mov Disord 2012;27:1718-28.

40 Brefel-Courbon C, Payoux P, Thalamas C, et al. Effect of levodopa on pain threshold in Parkinson's disease: a clinical and positron emission tomography study. Mov Disord 2005;20:1557-63.

41 Saint-Cyr JA, Trepanier LL, Kumar R, et al. Neuropsychological consequences of chronic bilateral stimulation of the subthalamic nucleus in Parkinson's disease. Brain 2000;123(Pt 10):2091-108.

42 Juri C, Rodriguez-Oroz M, Obeso JA. The pathophysiological basis of sensory disturbances in Parkinson's disease. J Neurol Sci 2010;289:60-5.

43 Pellaprat J, Ory-Magne F, Canivet $\mathrm{C}$, et al. Deep brain stimulation of the subthalamic nucleus improves pain in Parkinson's disease. Parkinsonism Relat Disord 2014;20:662-4. 